\title{
Phosphatidylcholine/cholesterol liposomes as vehicles for anthraquinone disperse dyes in wool dyeing
}

\author{
Ade la Maza, J L Parra, L Coderch and P Bosch
}

\author{
Centro de Investigación y Desarrollo, Consejo Superior de Investigaciones Cientificas, Calle Jorge Girona \\ 18-26,08034 Barcelona, Spain
}

The use $f$ liposomes containing increasing amounts of cholesterol as carriers for anthraquinone disperse dyes in the dyeing of wool fibres is described. Multilamellar lipid vesicles of egg phosphatidylcholine and containing Q Disperse Violet 1 at different phosphatidylcholine-cholesterol relative concentrations were used. Controlled exhaustion of dye on the wool was achieved, which was directly dependent on the liposome lipid concentration. Increasing the amount $\boldsymbol{f}$ cholesterol in the bilayers resulted in a decrease in dyebath exhaustion while improving the total amount $\boldsymbol{f}$ dye bonded to the wooi fibres. The dye's dispersion efficiency was also improved, compared with the use of conventional dispersing agents. The optimum application of these systems, both in terms of dyebath exhaustion and total amount of dye bonded to the wool samples, was directly correlated with the dye/ lipid weight ratio for the maximum level of dye encapsuiation efficiency.

\section{INTRODUCTION}

Over the last decade several investigations have been carried out into ways of reducing the degradation of wool fibres during conventional dyeing. The technology of microencapsulation has been one method adopted, in which the targeted slow release of protective agents can be introduced into the dyeing process [1]. Nevertheless, some technological difficulties related to the staining of wool with disperse dyes at high temperatures still exist, especially in the dyeing of wool/polyester blends [2]. Selecting appropriate disperse dyes and using suitable carriers are considered to be very important factors in preventing this problem $[3,4]$.

Merino wool fibres naturally contain $1 \%$ by weight of lipids, cholesterol $(\mathrm{CH})$ being one of the main components [5-7]. These iipids form the hydrophobic barrier of the celi membrane complex (CMC). Their structure is similar to that of the two lipid bilayers found in membranes of the keratinised stratum corneum of skin, which are capable of forming multiple biiayer structures [8]. The dyeing and diffusion properties of fibres are believed to be govemed by the lipid structure of the interceliular spaces that might act as 'solvents' for hydrophobic textile chemicals [9]. There is also evidence that dyes do, in fact, preferentially diffuse dong easily swolien regions such as the CMC (interceliular diffusion) rather than through the cuticle cells (transcellular diffusion) [10].

The possible application of liposomes as carriers in wool finishing is based on three main factors:

(a) The similarity between the bilayer structure of the CMC and that of the liposomes

(b) The important role played by the $\mathrm{CMC}$ in the processing of chemicals into the fibres

(c) The role of the hydrophobic interactions in the structural organisation of wool.
Thus liposomes made with pure phosphatidylcholine (PC) and containing lipids present in the $\mathrm{CMC}$ such as cholesterol have been used as vehicles for aqueous chlorine solutions in wool chlorination processes [11]. These applications result in an improvement in the homogeneity of these oxidative treatments, minimising wool degradation and enhancing the efficiency of subsequent treatments. Along these lines, the use of liposomes as carriers of commercially available milling acid dyes and disperse dyes in wool dyeing has been reported [12,13].

in the present work we studied the effects of including $\mathrm{CH}$ in lipid biiayers to obtain improved application in wool dyeing with disperse dyes. The physical stability of multilamellar liposomes (MLV) containing the anthraquinone dye CI Disperse Violet 1 at düferent PC:CH molar ratios was investigated, the dye concentration remaining constant. The application of these structures in dyeing untreated wool samples has also been examined, focusing on the kinetic aspects of dye adsorption and the dye-fibre bonding forces on wool fibres.

\section{EXPERIMENTAL}

Materials

Botany wool fabrics knitted from R64/2 tex (count 2128) yarns were used. Samples were Soxhlet extracted for $2 \mathrm{~h}$ with methylene chloride and rinsed with water purified by the Milli-Ro system (Millipore) and dried at room temperature.

Phosphatidylcholine was purified from egg lecithin (Merck) according to the method of Singleton [14], and shown to be pure by thin layer chromatography (TLC). Cholesterol was purchased from Sigma Chemical Co. (St. Louis, USA). Lipids were stored in chloroform under nitrogen at $-20^{\circ} \mathrm{C}$ until used. 
The disperse dye Oracetblau 2R, (Merck, CI Disperse Violet 1) was used; its chemical structure is given in Figure 1. This dye was selected as being a typical anthraquinone disperse dye, sparingly soluble in water $\left(0.3 \mathrm{mg} /\right.$ at $\left.25^{\circ} \mathrm{C}\right)$ and needing a high concentration of surface-active agent to be dispersed (17 mg/l in 1\% sodium oleyl-p-anisidide suiphonate solution (Lissapol LS, ICI) [15].<smiles>Nc1ccc(N)c2c1C(=O)c1ccccc1C2=O</smiles>

Figure 1 Chemical structure of the $\mathrm{Cl}$ Disperse Violet 1

The nonionic surfactant Triton X-100 (octylphenol with ten units of ethylene oxide, $100 \%$ active matter) was specially prepared by Tenneco SA (Barcelona, Spain). Polycarbonate membranes of 400 and $800 \mathrm{~nm}$, and membrane holders used for liposome extrusion, were purchased from Nucleopore (Pleasanton, USA).

\section{Preparation of MLV liposomes}

Multilamellar vesicles of a defined size $(400 \mathrm{~nm})$, and different lipid concentrations (1.25-3.0 mmol/ $)$, containing different concentrations of $\mathrm{CH}$ ( $\mathrm{PC}: \mathrm{CH}$ molar ratios from 19:1 to 4:1) were prepared following a method described by Bangham [16]. The dye concentration was held constant at $1.0 \mathrm{mmol} / \mathrm{l}$.

Afilm was formed by removing the organic solvent from a solution of egg $\mathrm{PC} / \mathrm{CH} /$ dye in chloroform/methanol (2:1) by rotary evaporation in a nitrogen atmosphere and low vacuum $47 \mathrm{kPa}(350 \mathrm{~mm} \mathrm{Hg})$. An aqueous phase containing $5 \%$ sodium sulphate and acetic acid (pH 5.5) was added to the film formed. The solutions were then swirled to remove the lipid from the walls of the flask and to disperse large lipid/dye aggregates; glass beads were added to facilitate dispersion. The resulting milky suspension were vortexed for $5 \mathrm{~min}$ and sonicated for $15 \mathrm{~min}$ at $30^{\circ} \mathrm{C}$ and $75 \mathrm{~W}$ (Labsonic 1510 B, Braun). Liposome suspensions were extruded through 800 and $400 \mathrm{~nm}$ polycarbonate membranes to obtain a uniform size distribution [17]. After preparation the resulting liposome suspensions were left to equilibrate for $15 \mathrm{~min}$ and immediately used in wool dyeing.

\section{Dyeing procedure}

Knitted wool samples were heated in freshly prepared MLV liposome suspensions under the conditions mentioned above. The dye was applied at a liquor ratio of $60: 1$ dong with 5\% o.w.f. anhydrous sodium suiphate; acetic acid was added to adjust the $\mathrm{pH}$ to 5.5. Dyeing in a MultiMat (Renigal) dyeing machine was started at $50^{\circ} \mathrm{C}$ and the temperature was raised by $0.9 \mathrm{deg} / \mathrm{min}$ to $90^{\circ} \mathrm{C}$ and dyeing continued for $120 \mathrm{~min}$. Samples were then rinsed with water for $10 \mathrm{~min}$ and dried at room temperature.
Dyebath exhaustion was determined using a Shimadzu UV-265FW spectrophotometex Liposome aliquots $(0.5 \mathrm{mi})$ were penodically added to quartz cuvettes filled with $2 \mathrm{ml}$ aqueous solution of Triton X-100 (20 g/), supplemented with sodium sulphate (5\%).The interaction between the nonionic surfactant Triton X-100 and liposome structures resulted in solubiisation of the lipid vesicles via the formation of mixed micelles [18,19], turning the liposome suspension into a clear solution.

Figure 2 shows the effect of the cleavage of liposome vesides by Triton X-100 on the absorption spectra of the dye at different PC: $\mathrm{CH}$ molar ratios. It can be seen that the $\lambda_{\max }$ value of the dye used in this study did not change in the presence of increasing amounts of $\mathrm{CH}$ in the lipid/ surfactant mixed micelles.

\section{Vesicle size distribution, dispersion efficiency and aggregation}

Mean veside size and the polydispersity of the liposome preparations were determined by a Photon correlator spectrometer (Malvem Autosizer 4700c PS/MV). Vesicle size distribution was studied by particle number measurement. Samples were adjusted to the appropnate concentration range. The measurements were made at $25^{\circ} \mathrm{C}$ with a detection angle of 909

The maximum amounts of dye dispersed with the MLV liposomes was defined as the weight ratio between the dispersed dye and the lipid in the iiposomes, $K$, and was determined spectrophotometrically. After preparation, liposome suspensions were left to equilibrate for $12 \mathrm{~h}$. Vesicle suspensions were then spun at $5000 \mathrm{rev} / \mathrm{min}$ for $15 \mathrm{~min}$ at $25^{\circ} \mathrm{C}$ in order to remove the unencapsuiated dye. Finally the concentration of dispersed dye was evduated by spectrophotometry after the destruction of the supematant lipid bilayers by adding Triton X-100 [18,19].

The aggregation state of the vesides was estimated to provide a measure of the physical stabiity of the liposome suspensions. This was done by monitoring the variation in vesicle size distribution of the liposome suspensions as a function of time.

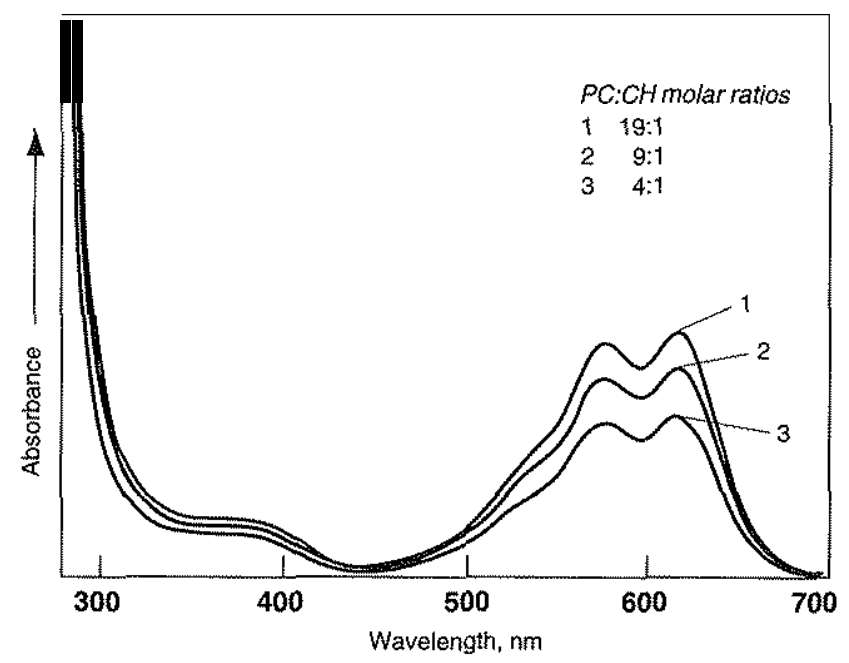

Figure 2 Absorption spectra of $\mathrm{Cl}$ Disperse Violet 1 in the presence of lipid/Triton $\mathrm{x}-100$ mixed micelles at different bilayer lipid compositions 
Billayer lipid composition

The lipid composition of different liposome vesides studied was determined using an Iatroscan MK-5 TLC-FID analyser. Coupling TLC to an automated detection system based on flame ionisation detection is a recent innovation which has considerably improved the sensitivity of TLC and allows quantitative estimation of separated materials. This method has been used to quantify many kinds of lipids from different sources [20]. This procedure was chosen in the present study to quantify lipid mixtures, even when they were forming liposomes in aqueous solutions, on the basis of previous experience of lipid analysis of keratinised tissues such as wool $[21,22]$.

\section{Dye extraction}

The dye superficiaily bonded to the fibres by non-polar forces (hydrophobic interactions, van der Waals forces and hydrogen bonds) was extracted with pure ethanol for $60 \mathrm{~min}$ at $25^{\circ} \mathrm{C}$ [12]. Subsequent extradions with ammonia solution $\left(0.5 \%\right.$ for $15 \mathrm{~min}$ at $\left.60^{\circ} \mathrm{C}\right)$ stripped dye that had diffused into the fibre and bonded ionically [15]

\section{RESULTS AND DISCUSSION}

\section{Dispersion efficiency of liposomes}

The variation in the total amount of dye dispersed in the presence of MLV liposomes at different biiayer compositions as a function of bilayer lipid concentration is indicated in Figure 3. A linear dependence was established in all cases. The slope of the straight lines obtained represents the weight ratio between dye and lipid, $K$. The $K$ values for each biiayer composition and the regression coefficients for each straight line are given in Table 1 . The $K$ values decreased as the $\mathrm{CH}$ concentration in the biiayers increased, reaching a minimum value at the $4: 1 \mathrm{PC}: \mathrm{CH}$ molar ratio $(K 0.18)$. The use of $\mathrm{PC} C \mathrm{CH}$ liposomes always resulted in a large increase in dye dispersion efficiency, compared with that of the conventional dispersing agent normally used for this dye $[13,15]$. Thus the optimum application of these systems (PC:CH 19:1 molar ratio) reduced the lipid concentration needed to disperse the same amount of dye by more than 140 times. At PC:CH molar ratios of $9: 1$ and $4: 1$ this reduction was about 117 and 105 times respectively. In practical dyeing this improvement could be reflected in lower process costs and lower dye concentrations

\section{Stability of liposome suspensions}

The possible aggregation of liposomes during dyeing was monitored by measuring the variations in the mean veside size and polydispersity indices of these suspensions using a quasi-elastic light scattering method [23]. The results obtained for liposome suspensions (lipid concentration 20 $\mathrm{mmol} / \mathrm{l}$ ) at different bilayer compositions and using the $K$ ratios for the maximum encapsulation efficiency of each system (Table 1)are given in Figure 4.

There was a small decrease in the mean particle size during the initial stage of dyeing, with a marked increase in polydispersity index. After $45 \mathrm{~min}$ both parameters increased progressively, reaching values of about $425-438$ nm and $0.13-0.15$ respectively after treatment. Increasing

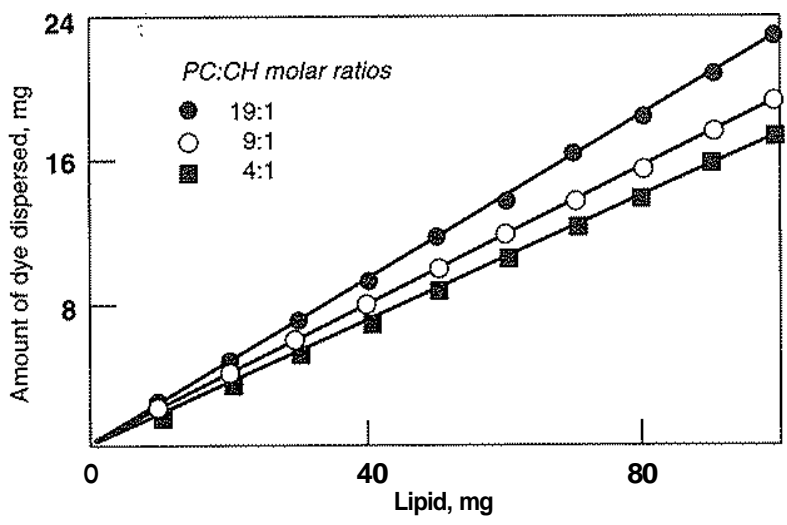

Figure 3 Maximum amounts of dispersed dye as a function of lipid concentrationfor three levels of $\mathrm{CH}$ in bilayers

Table 1 Weight dyellipid ratios $(K)$ and regression coefficientsof straight lines corresponding to the maximum dispersion efficiency of MLV liposomes

\begin{tabular}{lll}
$\begin{array}{l}\text { PC:CH } \\
\text { moiar ratio }\end{array}$ & $\mathbf{K}$ & $\mathbf{r}^{2}$ \\
\hline $19: 1$ & 0.24 & 0.994 \\
$9: 1$ & 0.20 & 0.993 \\
$4: 1$ & 0.18 & 0.992 \\
\hline
\end{tabular}

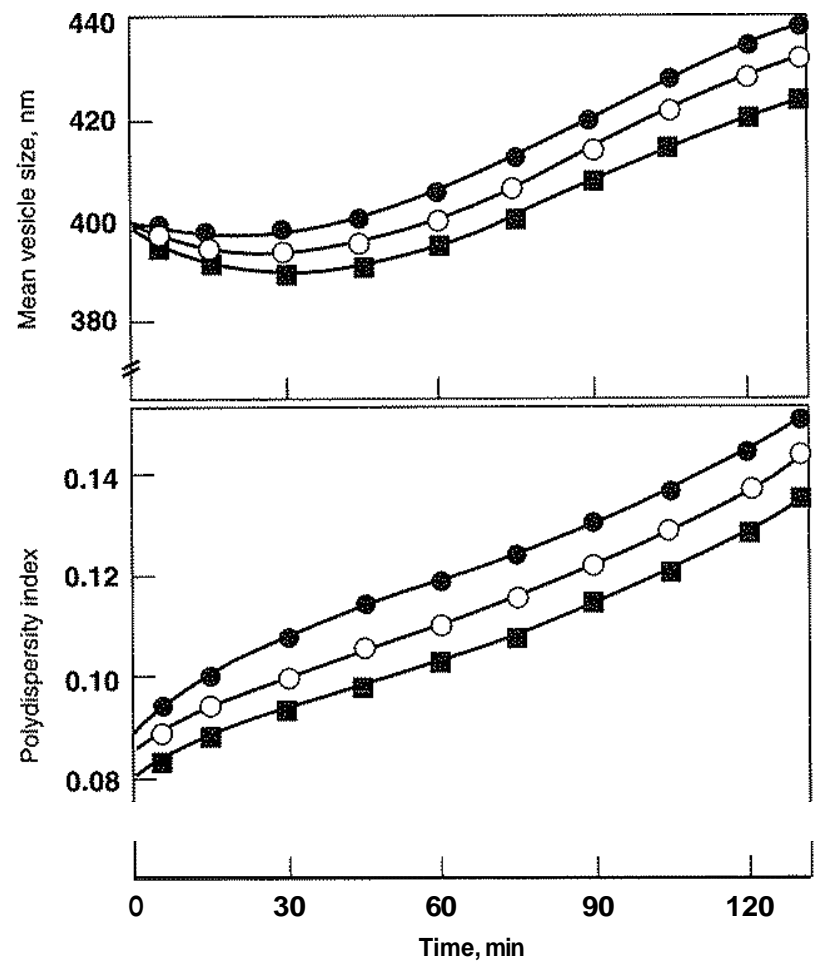

Figure 4 Mean vesicle size and polydispersity of MLV liposomes at different lipid compositions and $2 \mathrm{mmol} / \mathrm{i}$ total lipid concentration during the dyeing process (for key see Figure 3 ) 
amounts of $\mathrm{CH}$ in the biiayers enhanced the stabii of these structures with respect to aggregation, reducing the variations in both the mean particle size and the polydispersity index during dyeing. This behaviour is in agreement with the results reported by Scherphof et al. in studies on liposome stability [2A]. Furthermore, the mean veside size was maintained at around $400 \mathrm{~nm}$ and the polydispersity index below 0.24 for more than $24 \mathrm{~h}$ after preparation.

\section{Dyeing kinetics}

Kinetic studies of dyebath exhaustion on untreated wool samples in the presence of MLV liposomes were carried out, varying both the bilayer composition and the total lipid concentration, at constant dye concentration. The results obtained for the PC:CH molar ratio 19:1 are plotted in Figure 5. The exhaustion increased as the bilayer lipid concentration rose, reaching a maximum for $1.75 \mathrm{mmol} / \mathrm{l}$. Above this concentration, increasing amounts of lipids in bilayers retarded the rate of dyeing (minimum exhaustion for $3.0 \mathrm{mmol}$ was $69.1 \%$ ).A similar pattern in dyeing rates was observed at all the PC:CH molar ratios studied, although the lipid concentration needed to achieve the maximum exhaustion rose as the $\mathrm{CH}$ concentration in the biiayers increased. Thus, whereas the liposomes formed at the PC:CH molar ratio of 9:1 gave a maximum exhaustion at $2.25 \mathrm{mmol} / 1(79.2 \%)$, those formed by $4: 1$ needed 2.50 mmol/ lipid concentration to achieve this maximum $(73.5 \%$ )(results not plotted). It is interesting to note that the weight ratio $\mathrm{K}$ for the maximum exhaustion was always similar to that for the maximum encapsdation efficiency.

Figure 6 shows the exhaustion of various dye/liposome systems on wool samples at different lipid bilayer compositions, in all cases using the lipid concentration to achieve maximum dyebath exhaustion. It is noteworthy that increasing amounts of $\mathrm{CH}$ in bilayers resulted in a decrease in exhaustion on wool throughout the dyeing process. This effect was considered an important factor in

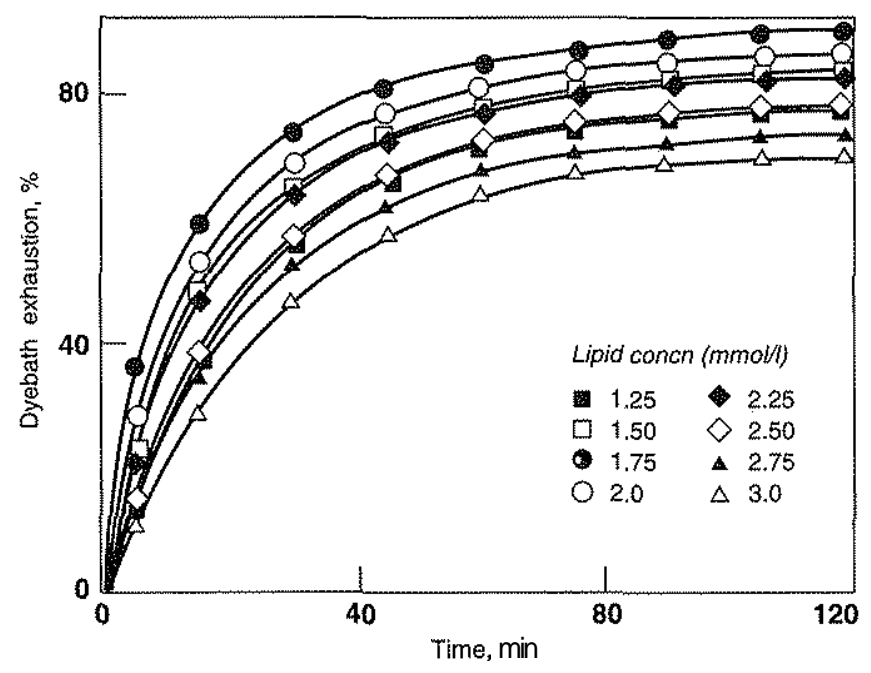

Figure 5 Exhaustion rates of $\mathrm{Cl}$ Disperse Violet 1 on untreated wool sarnples during dyeing in the presence of MLV liposomes at different bilayer lipid concentrations

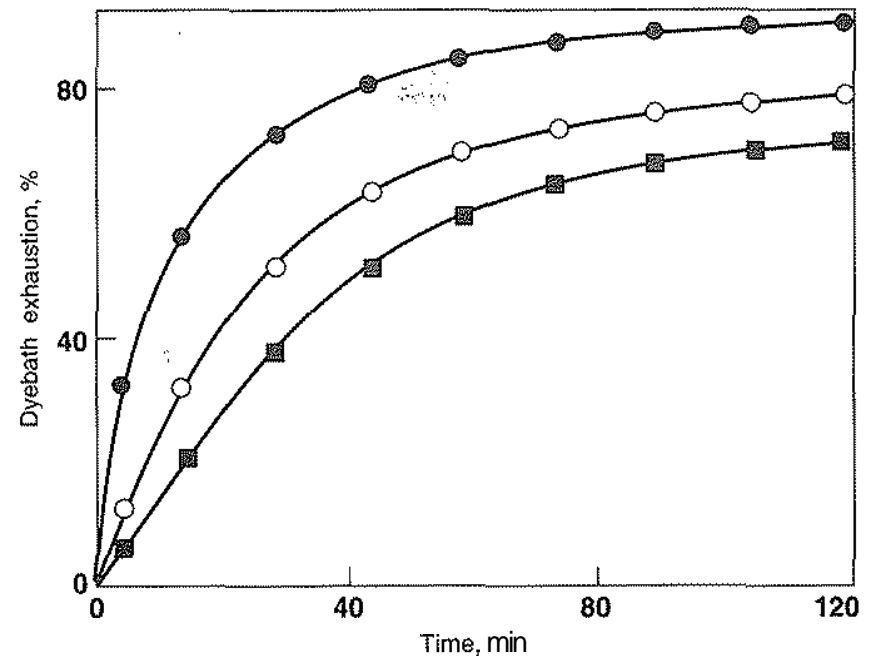

Figure 6 Exhaustion rates of $\mathrm{Cl}$ Disperse Violet 1 on untreated wooi sarnples during dyeing in the presence of MLV liposomes at different $\mathrm{PC}$. CH rnolar ratios (for key see Figure 3)

controlling the dyeing kinetics and improving the regularity of dye distribution in the wool fibres. Optical microscope observations of dyed samples provided evidence of a regulardye distribution throughout the fibres, this improvement being especially marked for the lipid concentrations corresponding to the maximum exhaustion. Furthermore, increasing amounts of $\mathrm{CH}$ in bilayers also resultedin an enhancement in dye distribution.

Influence of lipid bilayer composition on dye bonding in order to determine whether, as dye carriers, the lipid bilayers (containing increasing amounts of $\mathrm{CH}$ ) caused changes to dye-fibre bonding forces after dyeing, dye was extracted from the samples using pure ethanol [12] and ammonia [15]. The results obtained for different concentrations of $\mathrm{CH}$ in the liposomes are given in Tables $2-4$. In general ethanol extracted larger amounts of dye than did the ammonia solutions. This was because of the high solubility of the dye in ethanol and the presence of dye superficially bonded to the fibres by non-polar forces. However, the fact that very small amounts of dye were extracted via ammonia could be attributed to the important contribution of the non-polar forces in the dye-fibre bonding. These interactions, especially those that are hydrophobic in nature, may play an important role in dyefibre bonding inside the fibre. Furthermore, as the lipid bilayer concentration increased, the amounts of dye extracted from the samples using ethanol decreased. The amounts of extracted dye were, for each lipid concentration, inversely proportional to the $\mathrm{CH}$ concentration present in the biiayers. Ammonia extractions showed very low values in all cases.

Tables $2-4$ also show the total amount of bonded dye on the wool samples, as determined by Eqn 1:

$$
C_{b}=\frac{C_{a}-C_{e}}{C_{a}} \times 100
$$

where $C_{b}$ is the relative amount of bonded dye $(\%), C_{a}$ is the 
amount of absorbed dye (mg per $\mathrm{g}$ wool) and $C_{\mathrm{e}}$ is the total amount of extracted dye (mg pergwool).

In general, the higher the lipid concentration and the relative concentration of $\mathrm{CH}$ in the bilayers, the higher the amount of bonded dye. An exception was observed at the 19:1 PC:CH molar ratio, where the maximum amount of bonded dye was obtained at the $K$ ratio corresponding to the maximum exhaustion.

Figure 7 was obtained by plotting the amounts of bonded dye on the wool samples (expressed as differences between the amounts of adsorbed dye and total dye extracted) versus iipid concentration for three levels of $\mathrm{CH}$ in the bilayers. The maximum amount of bonded dye (points A, B and C) occurred at $\mathrm{K}$ ratios corresponding to the maximum levels of both the dyebath exhaustion and

Table 2 Amounts of dye adsorbed by, extractedfrom and bonded to wooi after dyeing in the presence of MLV liposomes at different iipid concentrationsand a $\mathrm{PC}: \mathrm{CH}$ moiar ratio of 19:1

\begin{tabular}{|c|c|c|c|c|c|}
\hline \multirow{3}{*}{$\begin{array}{l}\text { Lipid } \\
\text { conen } \\
\text { (mmol/s) }\end{array}$} & \multicolumn{3}{|c|}{ Dye concn (mg per g wooi) } & \multirow{3}{*}{$\begin{array}{l}\text { Amount } \\
\text { of dye } \\
\text { bonded (\%) }\end{array}$} & \multirow[b]{3}{*}{ TM 193} \\
\hline & \multirow[b]{2}{*}{ Adsorbed } & \multicolumn{2}{|c|}{ Extracteda } & & \\
\hline & & $A$ & $\mathrm{~B}$ & & \\
\hline 1.25 & 14.6 & 5.18 & 0.018 & 64.3 & 2 \\
\hline 1.50 & 15.8 & 5.18 & 0.016 & 67.2 & $2-3$ \\
\hline 1.75 & 16.6 & 4.74 & 0.014 & 71.4 & $3-4$ \\
\hline 2.00 & 16.2 & 4.95 & 0.010 & 69.4 & 4 \\
\hline 2.25 & 15.5 & 4.80 & 0.011 & 68.9 & $3-4$ \\
\hline 2.50 & 14.6 & 4.54 & 0.009 & 68.9 & $3-4$ \\
\hline 2.75 & 13.8 & 4.33 & 0.009 & 68.6 & $3-4$ \\
\hline 3.00 & 13.0 & 4.14 & 0.009 & 68.1 & $3-4$ \\
\hline
\end{tabular}

a A Extracted by ethanol [12]

B Extracted by ammonia [15]

Table 3 Arnounts of dye adsorbed by, extractedfrom and bonded to wool afier dyeing in the presence of MLV iiposomes at difierent lipid concentrations and a $\mathrm{PC}: \mathrm{CH}$ molar ratio of $9: 1$

Dye concn (mg per g wool)

\begin{tabular}{|c|c|c|c|c|c|}
\hline \multirow{2}{*}{$\begin{array}{l}\text { Lipid } \\
\text { conen } \\
\text { (mmol/) }\end{array}$} & \multirow[b]{2}{*}{ Adsorbed } & \multicolumn{2}{|c|}{ Extracted ${ }^{a}$} & \multirow{2}{*}{$\begin{array}{l}\text { Amount } \\
\text { of dye } \\
\text { bonded (\%) }\end{array}$} & \multirow[b]{2}{*}{ TM 193} \\
\hline & & A & B & & \\
\hline 1.25 & 12.5 & 4.23 & 0.014 & 66.2 & $2-3$ \\
\hline 1.50 & 13.1 & 3.67 & 0.013 & 71.9 & $3-4$ \\
\hline 1.75 & 13.8 & 3.27 & 0.013 & 76.2 & $3-4$ \\
\hline 2.00 & 14.4 & 2.75 & 0.012 & 80.8 & 4 \\
\hline 2.25 & 14.9 & 2.53 & 0.011 & 83.0 & $4^{b}$ \\
\hline 2.50 & 13.5 & 1.83 & 0.009 & 86.4 & $4^{b}$ \\
\hline 2.75 & 12.3 & 1.16 & 0.008 & 90.5 & $4^{b}$ \\
\hline 3.0 & 11.2 & 0.75 & 0.006 & 93.2 & $4^{b}$ \\
\hline
\end{tabular}

a For key see Table 2

b Orhigher
Table 4 Amounts of dye adsorbed by, extracted from and bonded to wool after dyeing in the presence of MLV liposomes at different lipid concentrations and a $\mathrm{PC}: \mathrm{CH}$ rnolar ratio of $4: 1$

\begin{tabular}{|c|c|c|c|c|c|}
\hline \multirow{3}{*}{$\begin{array}{l}\text { Lipid } \\
\text { concn } \\
\text { (mmol/l) }\end{array}$} & \multicolumn{3}{|c|}{ Dye concn (mg per g wooi) } & \multirow{3}{*}{$\begin{array}{l}\text { Amount } \\
\text { of dye } \\
\text { bonded (\%) }\end{array}$} & \multirow[b]{3}{*}{ TM 193} \\
\hline & \multirow[b]{2}{*}{ Adsorbed } & \multicolumn{2}{|c|}{ Extracted $^{\mathrm{a}}$} & & \\
\hline & & $A$ & B & & \\
\hline 1.25 & 10.4 & 2.83 & 0.010 & 72.8 & $3-4$ \\
\hline 1.50 & 11.0 & 2.26 & 0.009 & 79.5 & $3-4$ \\
\hline 1.75 & 11.7 & 1.76 & 0.008 & 84.9 & 4 \\
\hline 2.00 & 12.3 & 1.15 & 0.008 & 90.6 & $4^{b}$ \\
\hline 2.25 & 12.9 & 0.581 & 0.007 & 95.4 & $4^{\mathrm{b}}$ \\
\hline 2.50 & 13.9 & 0.396 & 0.005 & 97.1 & $4^{b}$ \\
\hline 2.75 & 13.2 & 0.364 & 0.003 & 97.2 & $4^{b}$ \\
\hline 3.0 & 12.3 & 0.337 & 0.003 & 97.2 & $4^{b}$ \\
\hline
\end{tabular}

a For key see Table 2

b Orhigher

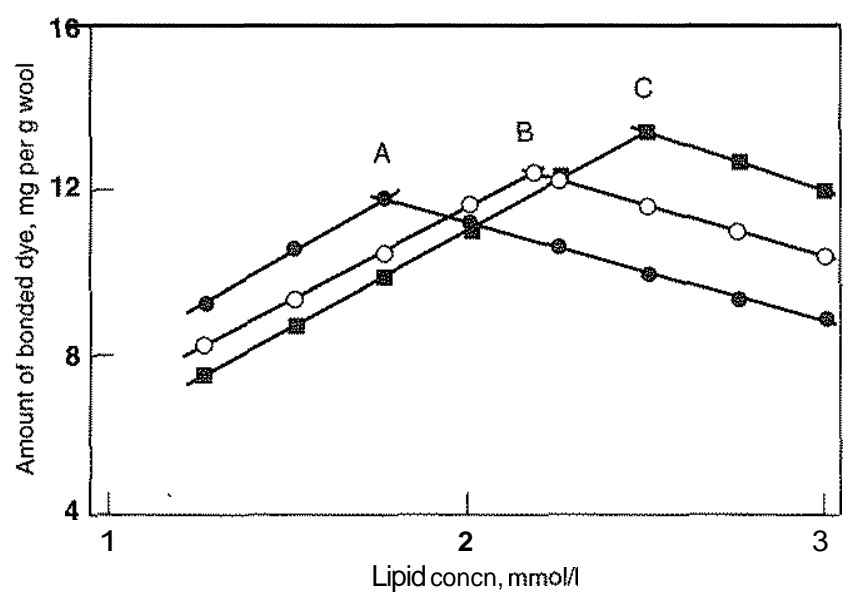

Figure 7 Arnounts of bonded dye in wool fibres versus iipid concentrationin liposomes fordifierent levels of $\mathrm{CH}$ in bilayers (for key see Figure 3 and text)

encapsdation effiàency of each system $(1.75,2.25$ and 2.50 $\mathrm{mmol} / \mathrm{l}$ for the PC:CH molar ratios 19:1, $9: 1$ and $4: 1$ respectively). It was interesting to note that the greatest amounts of bonded dye were obtained at the maximum $\mathrm{CH}$ concentration in the bilayers. This finding emphasises the role played by the $\mathrm{CH}$ in improving the dye-fibre bonding forces and justifies the incorporation of this component in the liposomes to obtain improved application in wool dyeing.

The wash fastness of dyed samples in the presence of liposomes was determined using the IWS TM 193 test method (corresponding to ISO 105:C06 (1978)and UNE 40120-81). The samples having the higher concentrations of bonded dye (Figure 7) also showed better levels of fastness (4 or higher).Thus, in generai, the higher the amount of $\mathrm{CH}$ in the bilayers, the better the colour fastness of the dyed samples. The samples that showed the best wash fastness results aiso attained a good level of xenon arc iight fastness (6 or higher) (ISO/R 105 (V) Part 2a, corresponding to UNE 
40-187-73). The colour fasiness results for the dyed samples are given in Tables 24.

Two principal factors seem to explain the important role played by $\mathrm{CH}$ in the exhaustion of dyes onto wool and the subsequentbonding into the keratin structures:

(a) The effect caused by $\mathrm{CH}$ in reducing the permeability of liposomes and enhancing their stabiüty to aggregation [24]

(b) The presence of $\mathrm{CH}$ in the hydrophobic barrier of the CMC (structuraliy similar to the two lipid biiayers simüar found in liposomes), which governs the diffusion of dyes into the fibre.

The first factor could be correlated with the decrease in dyebath exhaustion caused by $\mathrm{CH}$ present in the bilayers, and the second factor is related to the improvement in the dye bonding forces in the dyeingprocess.

\section{CONCLUSIONS}

The use of phosphatidylcholine-cholesterol multilamellar liposomes as vehicles for the anthraquinone dye C Disperse Violet 1 in wool dyeing can be useful for:

(a) Controiling dye exhaustion on untreated wool samples

(b) Improving the dye-fibre bondingforces

(c) Improvingdye distribution on the wool fibres.

The dispersion efficiency of MLV liposomes with respect to this dye is inversely proportional to the concentration of $\mathrm{CH}$ in the biiayers, the minimum being obtained for the PC:CH molar ratio 4:1 (weightratio dye/lipid $\mathrm{K}=0.18$ ) and the maximum for the PC:CH molar ratio 19:1 $(K=0.24)$ in the present work. However, the use of PC:CH MLV $1 \mathrm{i}$ posomes resulted in a clear improvement in the dye dispersion efficiency compared with conventional dispersino narmt.

All the liposomes studied ${ }^{\text {were }}$ stable during the dyeing process at $\mathrm{pH}$ 5.5. The amounts of dye exhausted and dye bonded to untreated wool samples for each PC:CH dye/ liposome system were directly proportional to $K$. corresponding to the maximum encapsulation efficiency of the system. Increasing the amount of $\mathrm{CH}$ in the bilayers resulted in a decrease in dyebath exhaustion on untreated wool samples and an increase in the total amount of dye bonded. The samples with higher amounts of bonded dye also gave better wash fasiness results, and these samples also showed good light fasiness performance.

This work was supported by funds from Dirección General de Investigación Científica y Técnica (DGICYT), Spain, Proyecto No. PB91-0065). The authors acknowledge the expert technical assistance of MrGKnorring.

\section{REFERENCES}

1. G Nelson, Rev. Prog. Col.. 21 (1991) 72.

2. J Wang and H Ásnes, J.S.D.C.,107 (1991) 274.

3. IWang and H Asnes, J.S.D.C.,107 (1991) 314.

4. PR Brady, Reo prog. Col., 22 (1992) 58.

5. L Coderch and C Soriano, Tex. Res. J.s 63 (1993)369.

6. J Hemling and $\mathrm{H}$ Zahn, Proc. 7th int. Wool Text. Res. Conf, Tokyo, Vol. 1 (1985) 181.

7. DE Rivett, Wool Sci. Ret., 67 (1991)1.

8. DT Downing, J. Lipid Res, 33 (1992)30I.

9. J D Leeder, Wool Sci. Rev. 63 (1986)3.

10. J D Leeder et al., Proc, 7th int. Wool Text. Res. Conf., Fokyo, Vol. 5 (1985) 99 .

11. A de la Maza and J L P m , Text. Res. J., 63 (1993) 44.

12. A de la Maza et al, J.S.D.C., 108 (1992) $\$ 40$.

13. A de la Maza and J L Parra, J.S.D.C., 110 (1994)64.

14. W S Singleton et al, J. Am. Oil Chem. Soc, 42 (1965)53.

15. E R Trotman, Dyeing and chemical technology of textile fibres', 6 th Edn (High Wycombe, UK: Charles Griffin, 1984) chapter 12.

16. A D Bangham, M M Standish and J C Watking, J. Mol. Biol., 13 (1965) 238.

17. FSzoka et al., Biochim. Biophys. Acfa, 601 (1980) 559 .

18. A Helenius and K Simons, Biochim. Biophys. Acta, 415 (1975) 29

19. D Lichtenberg, J Robson and E A Dennis, Biochim. Biophys. Acta, 737 (1983) 285.

20. R G Ackman, C A Mclead and A K Banerjee, I. Planar Chromatog, 3 179901450

21. L Coderch and CSoriano, Text. Res. J., 63(1993) 369.

22. L Coderch et al, Text, Res. 1., 62 (1992)704.

23. C S Chong and K Colbow, Biochim. Biophys. Acta., 426 (1976)260.

24. G L Scherphof, J Damen and J Wilschut in Liposometechnology, Vol. 3, Ed. G Gregoriadis (Boca Raton, FL: CRC Press, 1986) chapter 14. 